\title{
Bandwidth Analysis of a p-m-n Si Photodetector
}

\author{
Mohammed Sh. Ahmed \\ Department of Electrical \\ Engineering \\ Al-Mustansiriyah University \\ Iraq, Baghdad
}

\author{
Yassir TH. AL-Tulaihi \\ Department of Electrical \\ Engineering \\ Al-Mustansiriyah University \\ Iraq, Baghdad
}

\author{
Haider TH. Salim ALRikabi \\ Department of Electrical \\ Engineering \\ Wasit University \\ Iraq, Wasit
}

\begin{abstract}
The aim of this study is the bandwidth analysis of a p- $\pi-n$ photodetector, three different junction areas $(0.008,0.014$, $0.02) \mathrm{mm}^{2}$ were used with $\pi$-layer width of $5 \mu \mathrm{m}$, and the $\pi$ layer width required to get maximum bandwidth is $3.4 \mu \mathrm{m}$. The results showed that the bandwidth increases with the decreasing of detector area, this is because when detector area decreases the junction capacitance also decreases. The obtained bandwidth is $7.8 \mathrm{GHz}$ at $\pi$-layer width of $5 \mu \mathrm{m}$, the required biasing voltage is $17.55 \mathrm{~V}$. The best bandwidth obtained is $9 \mathrm{GHz}$ at a $\pi$-layer width of $3.4 \mu \mathrm{m}$, an area of $0.008 \mathrm{~mm}^{2}$ and required biasing voltage is $11.934 \mathrm{~V}$. In this paper mathematical relations have been found to get bandwidth, maximum bandwidth, and the requirements to achieve it. The requirements include choosing values of, $\pi$ layer width, biasing voltage, electric field, and carriers velocity. The mathematical relations results are very close to the experimental results. The results are achieved with the aid of MATLAB programming tool version 8.5.0.1976013 (R2015a).
\end{abstract}

\section{Keywords}

$\mathrm{Si}, \mathrm{p}-\pi-\mathrm{n}$, Photodetector, Bandwidth, Photodiode.

\section{INTRODUCTION}

When the photodiode is reverse biased, it operates as a photodetector. The $\mathrm{p}-\pi-\mathrm{n}$ photodetector bandwidth represents the data rate that photodetector could achieve it. The photodetector bandwidth is specified experimentally. In 1995, Lee and Zeghbroeck used metal-semiconductor-metal (MSM) photodetector that achieved a bandwidth of $3 \mathrm{GHz}$ at a biasing voltage of $10 \mathrm{~V}$ [1]. In 1996, Ho and Wong utilized a trench structure silicon-on-insulator (SOI), the obtained bandwidth is $2.3 \mathrm{GHz}$ at a wavelength of $790 \mathrm{~nm} \mathrm{[2].} \mathrm{In} \mathrm{1998,} \mathrm{G.} \mathrm{W.}$ Neudeck et al., designated a resonant cavity enhancement (RCE) photodetector with an $\mathrm{SiO} 2 /$ polysilicon bottom mirror and a single-crystal silicon absorption layer grown epitaxially by merged epitaxial lateral overgrowth (MELO), the detector bandwidth is $5 \mathrm{GHz}$, corresponding to a biasing voltage of $48 \mathrm{~V}$ [3]. In 2011, A. Habibpoor et al., used one-dimensional (1-D) simulation program based on the drift-diffusion model and Discrete Fourier Transform (DFT) method is developed. The program numerically solves the time-dependent continuity equations for electrons and holes in a semiconductor device. The model simulates carrier concentrations and the impulse response of a GaAs MSM photodetector at a constant bias voltage. The simulation showed that for a smaller value of carrier lifetime, the response fall time decreases without significantly reducing the responsivity of the device [4]. In 2014, Y. Hu et al., utilized one-dimensional and two-dimensional simulations of the drift-diffusion equations to determine the physical origin of the saturation in a simple heterojunction p-i-n photodetector at room temperature. Incomplete ionization, external loading, impact ionization, and the Franz-Keldysh effect are all included in the model. The results showed that the impact ionization has a greater effect on theelectrons than it does on the holes. The results also showed that the hole velocity saturates slowly with increasing reverse bias, and the hole current makes a large contribution to the harmonic power at $10 \mathrm{~V}$. This result implies that decreasing the hole injection will decrease the harmonic power [5]. The influencing parameters on the bandwidth are determined by the different mechanisms that transport the photo-generated charges to the contacts. These mechanisms are mainly drift and diffusion. The response time of the photodetector is set by two different contributions mainly: the transit time and the RC time constant [6]. The vertical illuminated $p-\pi-n$ photodetector is shown in Figure 1.

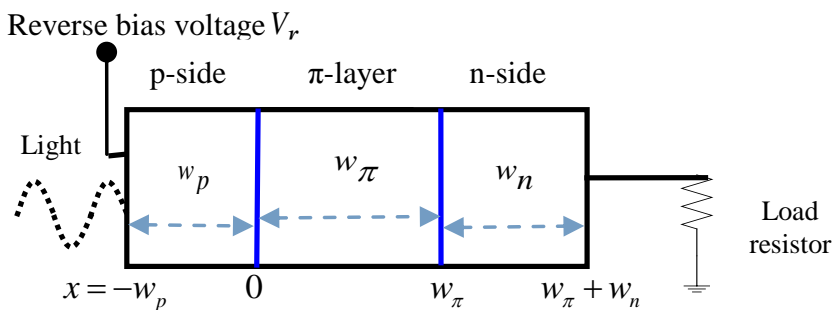

Figure 1: Vertically illuminated $\mathbf{p}-\pi-n$ photodetector

As shown from Figure 1, $w_{p}$ represents bulk p-side width $w_{n}$ is bulk n-side width, and $w_{\pi}$ is $\pi$-layer width. Assume that the incident light is coherent, so the photon energy $E_{p h}$ is [7],

$E_{p h}=h f$

where $\boldsymbol{h}$ is Planck's constant, and $\boldsymbol{f}$ is the frequency of incident photons.

\section{TRANSIT TIME LIMITED BANDWIDTH}

The transit time or drift time, is the time that the carriers need to passes through $\pi$-layer [8].

\subsection{Electrons}

The flow of electrons in $\pi$-layer is governed by [9],

$$
\frac{\partial n(x, t)}{\partial t}=\frac{1}{q} \frac{\partial J_{n}(x)}{\partial x}+\left[G_{n}(x, t)-U_{n}(x, t)\right]
$$

Where $n(x, t)$ is electrons concentration, $q$ is the carrier charge constant, $J_{n}(x, t)$ is electrons drift current density, $G_{n}(x, t)$ is electrons generation rate, and $U_{n}(x, t)$ is the electrons recombination rate. 
At the reverse biasing, high electric field $E$ generated within $\pi$-layer, so there is no recombination in it; therefore, neglecting the recombination term from Eq.(2) yields,

$\frac{\partial n(x, t)}{\partial t}=G_{n}(x, t)+\frac{1}{q} \frac{\partial J_{n}(x, t)}{\partial x}$

The electrons drift current density in $\pi$-layer is [10], $J_{n}(x, t)=q v_{s, n} n(x, t)$

where $v_{s, n}$ is electron saturation velocity. The Fourier transform to Eq.(4a) is,

$J_{n}(x, \omega)=q v_{s, n} n(x, \omega)$

Also from Eq.(4a),

$\frac{\partial J_{n}(x, t)}{\partial x}=q v_{s, n} \frac{\partial n(x, t)}{\partial x}$

Substituting into Eq. (3) yields,

$\frac{\partial n(x, t)}{\partial t}=G_{n}(x, t)+v_{s, n} \frac{\partial n(x, t)}{\partial x}$

The Fourier transform to Eq. (5a) is,

$j \omega n(x, \omega)=G_{n}(x, \omega)+v_{s, n} \frac{\partial n(x, \omega)}{\partial x}$

The electrons generation rate is given by,

$G_{n}(x, \omega)=G_{n}(0, \omega) e^{-\alpha \cdot x}$

Substituting into Eq. (5b) gives,

$v_{s, n} \frac{\partial n(x, \omega)}{\partial x}-j \omega n(x, \omega)=-G_{n}(0, \omega) e^{-\alpha x}$

The total solution to Eq.(6) is the sum of the homogeneous and particular solutions, $n_{h}(x, \omega)$ and $n_{p}(x, \omega)$, respectively, and is given by,

$n(x, \omega)=n_{h}(x, \omega)+n_{P}(x, \omega)$

\subsubsection{At Dark}

$G_{n}(0, \omega) e^{-\alpha x}=0$, so Eq. (6) reduced to,

$\frac{\partial n(x, \omega)}{\partial x}-\frac{j \omega n(x, \omega)}{v_{s, n}}=0$

The homogeneous solution to Eq.(6) represents the total solution to Eq. (8), which is,

$n_{h}(x, \omega)=C_{1} e^{j k x}$

where $C_{1}$ and $k$ are constants.

The derivative to Eq. (9a) is,

$\frac{\partial n_{h}(x, \omega)}{\partial x}=j k C_{1} e^{j k x}$

Substituting Eqs. (9a) and (9b) into Eq. (8) gives,

then substituting into Eq. (9a) yields,

$n_{h}(x, \omega)=C_{1} e^{\frac{j \omega x}{v_{s, n}}}$

\subsubsection{At Illumination}

The particular solution to Eq. (6) is,
$n_{P}(x, \omega)=C_{2} e^{-\alpha x}$

where $C_{2}$ is constant.

Substituting Eqs.(9c) and (10) into Eq.(7) obtains,

$n(x, \omega)=C_{1} e^{\frac{j \omega x}{v_{S, n}}}+C_{2} e^{-\alpha x}$

From Eq.(11a),

$\frac{\partial n(x, \omega)}{\partial x}=\frac{j \omega}{v_{s, n}} C_{1} e^{\frac{j \omega x}{v_{s, n}}}-\alpha C_{2} e^{-\alpha x}$

Substituting Eqs.(11a) and (11b) into Eq.(6) gives,

$C_{2}=\frac{G_{n}(0, \omega)}{j \omega+\alpha v_{s, n}}$

and then substituting into Eq.(11a) gets,

$n(x, \omega)=C_{1} e^{\frac{j \omega x}{v_{s, n}}}+\frac{G_{n}(0, \omega) e^{-\alpha x}}{j \omega+\alpha v_{s, n}}$

Using the boundary condition $n(0, \omega)=0$, then from Eq.(12a),

$C_{1}=\frac{-G_{n}(0, \omega)}{j \omega+\alpha v_{s, n}}$

Substituting $C_{1}$ into Eq.(12a), the total solution to the Eq.(6) is given by,

$n(x, \omega)=\frac{G_{n}(0, \omega)}{j \omega+\alpha v_{s, n}}\left[-e^{\frac{j \omega x}{v_{s, n}}}+e^{-\alpha x}\right]$

Substituting Eq.(12b) into Eq.(4b) gives,

$J_{n}(x, \omega)=\frac{q v_{s, n} G_{n}(0, \omega)}{j \omega+\alpha v_{s, n}}\left[-e^{\frac{j \omega x}{v_{s, n}}}+e^{-\alpha x}\right]$

\subsection{Holes}

The flow of holes in the $\pi$-layer is governed by [11],

$\frac{\partial p(x, t)}{\partial t}=-\frac{1}{q} \frac{\partial J_{p}(x)}{\partial x}+\left[G_{p}(x, t)-U_{p}(x, t)\right]$

where $p(x, t)$ is holes concentration, $J_{p}(x, t)$ is holes drift current density, $G_{p}(x, t)$ is holes generation rate, and $U_{p}(x, t)$ is holes recombination rate.

Due to high electric field in $\pi$-layer, so there is no recombination in this region, so neglecting the recombination term from Eq.(14) yields,

$\frac{\partial p(x, t)}{\partial t}=G_{p}(x, t)-\frac{1}{q} \frac{\partial J}{p^{(x, t)}}$

The hole drift current density in $\pi$-layer is [12],

$J_{p}(x, t)=q v_{s, p} p(x, t)$

where $v_{s, p}$ is hole saturation velocity.

The procedure used to find $J_{n}(x, \omega)$ is the same to finds $J_{p}(x, t)$ with the boundary condition $p\left(w_{\pi}, \omega\right)=0$, so hole drift current density is,

$J_{p}(x, \omega)=\frac{q v_{s, p} G_{p}(0, \omega) e^{-\alpha w}}{j \omega-\alpha v_{s, p}}\left[e^{-\alpha(x-w)}-e^{\frac{j \omega}{v_{s, p}}(w-x)}\right]$ 


\section{TOTAL CURRENT}

The total current density $J_{t o t}(x, t)$ is the sum of conduction current $\left[J_{p}(x, t)+J_{n}(x, t)\right]$, and the displacement current $[\varepsilon E(x, t)]$,

$J_{\text {tot }}(x, t)=\left[J_{p}(x, t)+J_{n}(x, t)\right]+\left[\varepsilon \frac{d E(x, t)}{d t}\right]$

where $\mathcal{E}$ is permittivity.

The Fourier transform to Eq.(17a) is,

$J_{\text {tot }}(x, \omega)=J_{p}(x, \omega)+J_{n}(x, \omega)+j \omega \varepsilon E(x, \omega)$

where $E(\omega)$ is the electric field harmonic component at $\boldsymbol{\omega}$.

When electrons exited from valence band to the conduction band, leaves behind it holes; therefore,

$G_{n}(x, \omega)=G_{p}(x, \omega)=G(x, \omega)$

The incident optical power $P_{o p t}$ is,

$P_{o p t}(t)=P_{o p t}(\omega) e^{j \omega t}$

and

$P_{o p t}(x, \omega)=\frac{h f A G(x, \omega)}{\alpha}$

The transit time, for electrons $\tau_{t r, n}$, and for holes $\tau_{t r, p}$, are [13],

$\tau_{t r, n}=\frac{w_{\pi}}{v_{s, n}}$

$\tau_{t r, p}=\frac{w_{\pi}}{v_{s, p}}$

Integrating both sides of Eq.(17b) from 0 to $w_{\pi}$ yields the total harmonic current $I_{t o t}(\omega)$

$$
\begin{aligned}
& I_{t o t}(\omega)=\alpha w_{\pi} \frac{q}{h f} P(0, \omega)\left\{\left(\frac{e^{-\alpha w_{\pi}}-1}{\alpha w_{\pi}\left(\alpha w_{\pi}-j \omega \tau_{t r, p}\right)}+\frac{\left(e^{j \omega \tau_{t r, p}}-1\right) e^{-\alpha w_{\pi}}}{j \omega \tau_{t r, p}\left(\alpha w_{\pi}-j \omega \tau_{t r, p}\right)}\right.\right. \\
& \left.+\frac{1-e^{-\alpha w_{\pi}}}{\alpha w_{\pi}\left(j \omega \tau_{t r, n}+\alpha w_{\pi}\right)}+\frac{1-e^{j \omega \tau_{t r, n}}}{j \omega \tau_{t r, n}\left(j \omega \tau_{t r, n}+\alpha w_{\pi}\right)}\right)+j \omega \frac{A \varepsilon}{w_{\pi}} V(\omega)
\end{aligned}
$$

Where the term in braces is the small-signal short-circuit photocurrent $-I_{L}(\omega)$, the last term is the current absorbed by $\pi$-layer geometric capacitance, $V$ is voltage drop across $\pi$ layer, and $A$ is junction area.

Eq.(20a) can be written as,

$I_{t o t}(\omega)=-I_{L}(\omega)+j \omega A C_{j} V(\omega)$

where $C_{j}$ is the junction capacitance.

\section{NORMALIZED RESPONSIVITY}

The normalized responsivity $\tau(\omega)$ of a $p-\pi-n$ photodetector is given by,

$$
\begin{aligned}
\tau(\omega) & =\frac{I_{L}(\omega)}{I_{L}(0)}=\frac{\alpha w_{\pi}}{\alpha w_{\pi}-j \omega \tau_{t r, p}}\left[\frac{1}{\alpha w_{\pi}}+\frac{1-e^{j \omega \tau_{t r, p}}}{j \omega \tau_{t r, p}\left(e^{-\alpha w_{\pi}}-1\right)}\right] \\
& -\frac{\alpha w_{\pi}}{\alpha w_{\pi}+j \omega \tau_{t r, n}}\left[\frac{1}{\alpha w_{\pi}}+\frac{1-e^{j \omega \tau_{t r, n}}}{j \omega \tau_{t r, n}\left(e^{-\alpha w_{\pi}}-1\right)}\right]
\end{aligned}
$$

The optimum design of a high-speed photodetector requires:

a. The photodetector should illuminated from the p-side. Since the minority carriers in the $\mathrm{p}$-side are electrons which have mobility larger than the mobility of holes in the n-side. So Eq.(21) reduce to,

$$
\tau(\omega)=-\frac{\alpha w_{\pi}}{\alpha w_{\pi}+j \omega \tau_{t r, n}}\left[\frac{1}{\alpha w_{\pi}}+\frac{1-e^{j \omega \tau_{t r, n}}}{j \omega \tau_{t r, n}\left(e^{-\alpha w_{\pi}}-1\right)}\right]
$$

b. The diffusion process is slow, which occurs in the $\mathrm{p}$ and $\mathrm{n}$ sides, to avoid it. The photodetector design should be such as $w_{\pi} \gg 1 / \alpha$ (where $\alpha$ is absorption coefficient) because most of light absorbed at $1 / \alpha$; therefore, Eq.(22) becomes,

$\tau(\omega) \approx\left|\frac{\sin \left(\frac{\omega \tau_{t r, n}}{2}\right)}{\frac{\omega \tau_{t r, n}}{2}}\right|$

The $3 \mathrm{~dB}$ bandwidth condition is

$20 \log _{10}\left|\tau\left(\omega_{3 \mathrm{~dB}, t r}\right)\right|=-3 \mathrm{~dB} \Rightarrow \frac{\omega_{3 \mathrm{~dB}, t r} \tau_{t r, n}}{2} \approx 1.391$

and

$\omega_{3 \mathrm{~dB}, t r}=2 \pi f_{3 \mathrm{~dB}, t r}$

where $f_{3 \mathrm{~dB}, t r}$ is transit time limited bandwidth, and it is given by substituting $\omega_{3 \mathrm{~dB}, t r}$ into Eq.(24),

$f_{3 \mathrm{~dB}, t r} \approx \frac{0.443 v_{s, n}}{w_{\pi}}$

Using Eq.(19a), Eq.(25a) becomes,

$f_{3 \mathrm{~dB}, t r} \approx \frac{0.443}{\tau_{t r, n}}$

\section{RC TIME LIMITED BANDWIDTH}

The equivalent circuit of a $p-\pi-n$ photodetector that includes all of circuit parameters is shown in Figure 2.

where $I_{d r i f t, p h}$ is the drift current through $\pi$-layer. The RC time constant is related with the device parameters that include junction capacitance $\left(C_{\pi}\right)$, diode resistance $\left(R_{j}\right)$, external load resistance $\left(R_{L}\right)$ and series resistance $\left(R_{S}\right)$ [14]. The series resistance is the bulk and the contact resistance and is often neglected due to being usually only a few ohms. The 
equivalent junction capacitance is,

$C_{j}=\frac{\varepsilon A}{w_{\pi}}$

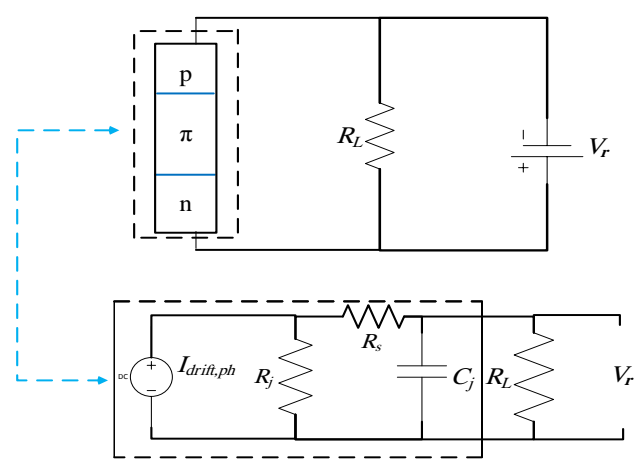

Figure 2: Equivalent electrical circuit of a p- $\pi-n$ photodetector.

The diode resistance can be found by Ohm's law as follows;

$R_{j}=\frac{V_{r}}{I_{d r i f t, p h}}$

The external load resistance represents the loading effects of any following circuit. The standard impedance $50 \Omega$ is chosen to be the load resistance. Therefore, the total resistance of the equivalent circuit $\left(R_{e q}\right)$ is given as,

$R_{e q}=\frac{\left(R_{s}+R_{j}\right) R_{L}}{\left(R_{s}+R_{j}\right)+R_{L}} \approx \frac{R_{j} R_{L}}{R_{j}+R_{L}}$, therefore,

$R_{e q} \approx R_{L}$

The photodetector capacitance also has significant influence on the overall response time via the RC time constant $\tau_{R C}$ which is defined as,

${ }^{\tau_{R C}}=R_{L} C_{j}=R_{L} \frac{\varepsilon A}{w_{\pi}}$

The RC time limited bandwidth $f_{3 \mathrm{~dB}, R C}$ is [15],

$$
f_{3 \mathrm{~dB}, R C} \approx \frac{1}{2 \pi \tau_{R C}}
$$

\section{BANDWIDTH}

The RC time and transit time are independent parameters, so the total equivalent time $T$ is,

$$
\begin{aligned}
T^{2} & =\tau_{t r, n}^{2}+\tau_{R C}^{2} \quad \text { so, } \\
B^{2} & =f_{3 \mathrm{~dB}, t r}^{-2}+f_{3 \mathrm{~dB}, R C}^{-2}
\end{aligned}
$$

where $\boldsymbol{B}$ is bandwidth

Substituting Eqs.(25b) and (30) into Eq.(31) gets,

$$
B=\frac{0.443}{\left[\tau_{t r, n}^{2}+\left(2.78 \tau_{R C}\right)^{2}\right]^{0.5}}
$$

\section{CARRIERS VELOCITY}

Carriers velocity are given by this experimental relation [16],

$$
v_{s, n-s, p}=\frac{v_{s}}{\left[1+\left(E_{o} / E\right)^{\gamma}\right]^{1 / \gamma}}
$$

where $v_{s}$ is the saturation velocity $\left(10^{7} \mathrm{~cm} / \mathrm{s}\right.$ for $\mathrm{Si}$ at $\left.300 \mathrm{~K}\right)$, $E_{O}$ is a constant $\left(7 * 10^{3} \mathrm{~V} / \mathrm{cm}\right.$ for electrons and $2 * 10^{4} \mathrm{~V} / \mathrm{cm}$ for holes), and $\gamma$ is 2 for electrons and 1 for holes.

\section{MAXIMUM BANDWIDTH}

The maximum bandwidth can be obtained by taking partial differentiate to Eq.(32) with respect to $\tau_{t r n}$,

$$
\frac{\partial B}{\partial \tau_{t r, n}}=\frac{-0.443 * 0.5 *\left[\tau_{t r, n}^{2}+\left(2.8 \tau_{R C}\right)^{2}\right]^{-0.5 *\left(2 . \tau_{t r, n}\right)}}{\left[\tau_{t r, n}^{2}+\left(2.78 . \tau_{R C}\right)^{2}\right]}
$$

Setting the equation above to zero to find $\tau_{t r, n}$ that gives maximum bandwidth, yields,

$\tau_{t r, n}=2.8 * \tau_{R C}$

Eq.(34) represents the condition to get maximum bandwidth. The value of $w_{\pi}$ at which maximum bandwidth is obtained can be found by substituting Eqs.(19a) and (29) into Eq.(34), $w_{\pi}=1.2145 * 10^{-5} \sqrt{v_{s, n} A}$

The flowchart in Figure 3, showing the steps to obtain the bandwidth at any wavelength of incident light to the photodetector. 


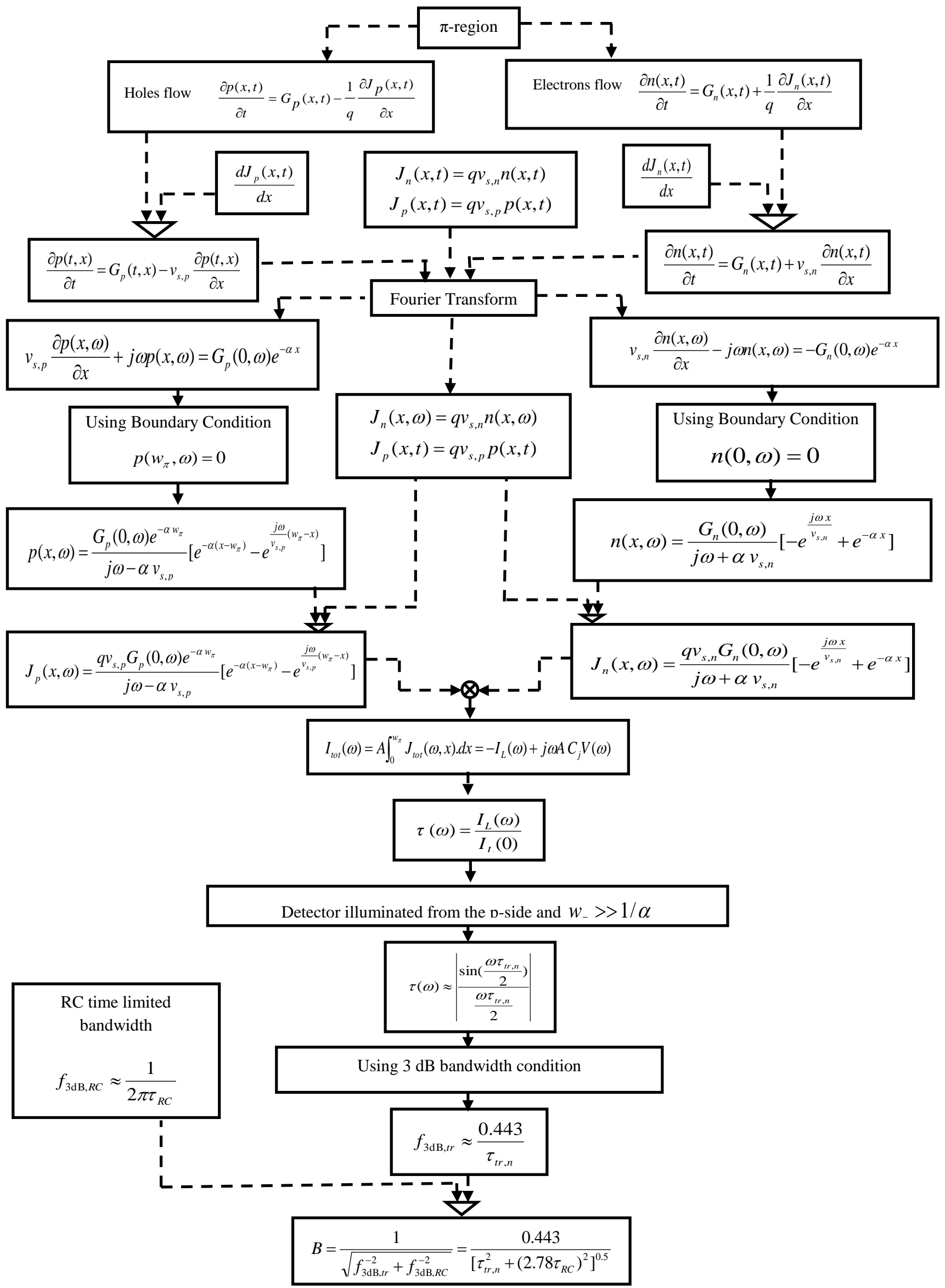

Figure 3: Flowchart showing the steps to obtain the bandwidth. 


\section{RESULTS AND DISCUSSIONS}

The software tool for the results obtained is a MATLAB version 8.5.0.197613 (R2015a) program. The carriers velocity is a function of electric field due to Eq.(33) as shown in Figure 4. The electron velocity is larger than hole velocity. At a field of about $3.51 * 10^{4} \mathrm{~V} / \mathrm{cm}$, the obtained electron velocity is $9.8^{*} 10^{6} \mathrm{~cm} / \mathrm{s}$, and hole velocity is $6.37 * 10^{6} \mathrm{~cm} / \mathrm{s}$.

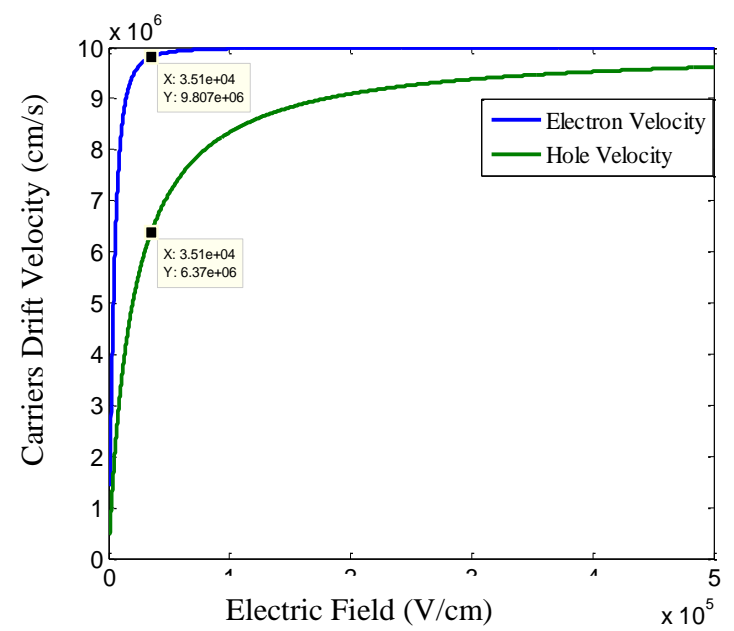

Figure 4: Carriers drift velocity versus electric field.

The bandwidth for different junction areas is shown in Figure 5 , the best bandwidth obtained is at area of $0.008 \mathrm{~mm}^{2}$.

Choosing $\pi$-layer width of $5 \mu \mathrm{m}$, at this width, the biasing voltage required is $V_{r}=E w_{\pi}==3.51 * 10^{4} * 5 * 10^{-4}=17.55 \mathrm{~V}$. Substituting the obtained electron velocity and the junction area of $0.008 \mathrm{~mm}^{2}$ into Eq.(35), the value of $\pi$-layer width at which maximum bandwidth occurs is $3.4 \mu \mathrm{m}$ as shown in Figure 5, the biasing voltage at which maximum bandwidth obtain is $V_{r}=E w_{\pi}=3.51 * 10^{4} * 3.4 * 10^{-4}=11.934 \mathrm{~V}$.

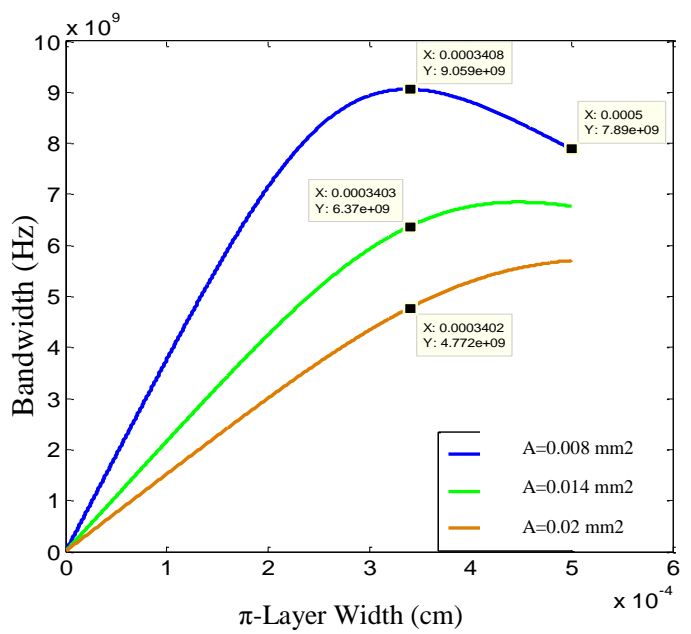

Figure 5: Bandwidth versus intrinsic layer width fordifferent junction areas.

The transit time limited bandwidth due to Eq.(25b) and RC time limited Bandwidth due to Eq.(30) are shown in Figure 6, at $w_{\pi}=3.4 \mu \mathrm{m}$ the maximum bandwidth obtained at this point due to Eq.(35). So as shown in Figure 5, when $w_{\pi}$ less than $3.4 \mu \mathrm{m}$ the bandwidth is directly proportional to $w_{\pi}$ since $\mathrm{RC}$ time limited bandwidth is dominated, but when $w_{\pi}$ larger than
$3.4 \mu \mathrm{m}$, the bandwidth is inversely proportional to $w_{\pi}$ since the transit time limited bandwidth is dominated.
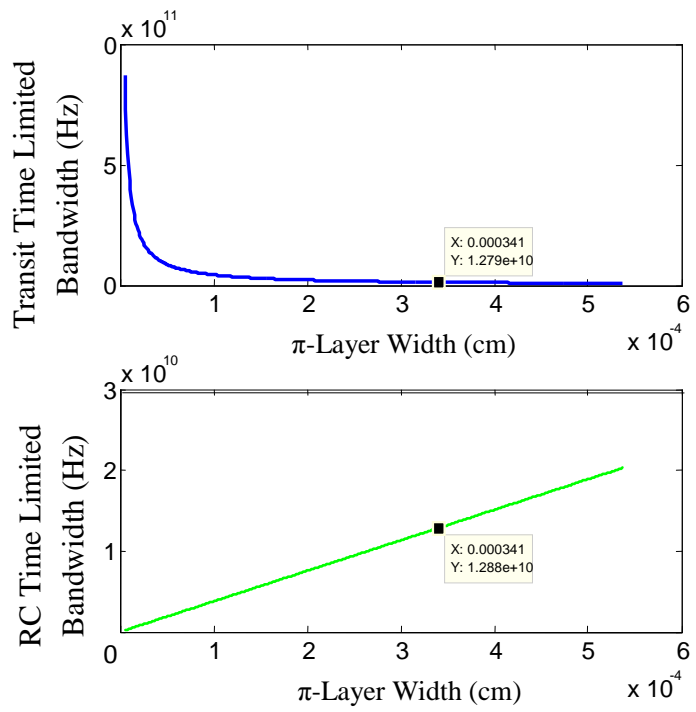

Figure 6: Transit and RC times limited bandwidth.

\section{CONCLUSIONS}

The optimum design of a high-speed photodetector requires, elimination of the diffusion currents, elimination of parasitic effects, and equalization of the transit time limited bandwidth with the RC time limited bandwidth such as $\tau_{t r, n}=2.8^{*} \tau_{R C}$. The bandwidth is unaffected by the absorption coefficient. The bandwidth of the vertically illuminated photodetector is affected mainly by $\pi$-layer width.

The Future work can be extended for vertical illuminated $\mathrm{p}-\pi-\mathrm{n}$ photodetector is that solving three-dimensional electrons and holes continuity equations, then follow the steps as shown in Figure 3 to get three-dimensional bandwidth of a $\mathrm{p}-\pi$-n photodetector.

\section{ACKNOWLEDGMENTS}

I would like to thanks to guide, Dr. Muneer A. Hashem who guided with great expertise and who supported on each time to development this paper.

\section{REFERENCES}

[1] H. C. Lee, and B. V. Zeghbroeck, "A novel high-speed silicon MSM photodetector operating at $830 \mathrm{~nm}$ wavelength," IEEE Electron Device Letters, vol. 16, no. 5, pp. 175-177, May 1995.

[2] J. Y. L., Ho, and K. S. Wong, "High-speed and highsensitivity silicon-on-insulator metal-semiconductormetal photodetector with trench structure," Applied Physics Letters, vol. 69, no. 1, pp. 16-18, May 1996.

[3] G. W. Neudeck, J. Denton, J. Qi, J. D. Schaub, R. Li, and J. C. Campbell, "Selective epitaxial growth Si resonantcavity photodetector," IEEE Photonics Technology Letters, vol. 10, no. 1, pp. 129-131, January 1998.

[4] A. Habibpoor and H. R. Mashayekhi, "Numerical modeling of the transient response of metalsemiconductor-metal photodetector using discrete Fourier transform method," Journal of Physics, vol. 286, no. 1, pp. 1-6, 2011. 
[5] Y. Hu, B. S. Marks, C. R. Menyuk, V. J. Urick and K. J. Williams, "Modeling Sources of Nonlinearity in a Simple p-i-n Photodetector," Journal of Lightwave Technology, vol. 32, no. 20, pp. 3710-3720, April 2014.

[6] J. P. Colinge, and C. A. Colinge, "Physics of semiconductor devices," Springer, 2005.

[7] SasaRadovanovic, Anne Johan Annema, and Bram Nauta, "High-speed photodiodes in standard CMOS technology," Springer, 2006.

[8] Safa O. Kasap, "Optoelectronics \&photonics," $2^{\text {nd }}$ Ed., Prentice Hall, 2012.

[9] B. Van Zeghbroeck, "Principles of semiconductor devices," Prentice Hall, 2001.

[10] Donald A. Neamen, "Semiconductor physics and devices," $3^{\text {rd }}$ Ed., McGraw-Hill, 2003.

[11] Chuang Shun Lien, and Shun L. Chuang, "Physics of optoelectronic devices," John Wiley \& Sons, 1995.

[12] Zhicai He, ChengmeiZhong, Xun Huang, Wai Yeung Wong, Hongbin Wu, Liwei Chen, Shijian Su, and Yong
Cao, "Simultaneous enhancement of open-circuit voltage, short-circuit current density, and fill factor in polymer solar cells," dvanced Materials, vol. 23, no. 40, pp. 4636-4643, Oct. 2011.

[13] Chen, Jau Wen, Dae Kaen Kim, and Mukunda B. Das. "Transit-time limited high-frequency response characteristics of MSM photodetectors," Electron Devices, IEEE Transactions, vol. 43, no. 11, pp. 18381843,1996

[14] S. M. Sze, and Kwok K. NG, "Physics of Semiconductor Devices," 3rd Ed., John Wiley \& Sons, 2007.

[15] B. Gao, H. Wang, C. Y. Liu, Q. Q. Meng, Y. Tian, K. S. Ang, and J. H. Si, "Design and analysis of InP-based waveguide uni-traveling carrier photodiode integrated on silicon-on-insulator through $\mathrm{Al} 2 \mathrm{O} 3$ bonding layer," IEEE Photonics Journal, vol. 6, no. 5, Aug. 2014.

[16] Rogalski A., "Fundamentals of infrared detector technologies," $2^{\text {nd }}$ Ed., CRC Press, 2010. 